\title{
Philosophiques
}

\section{Phénoménologie et dialectique du travail}

\section{Douglas Moggach}

Volume 15, numéro 2, automne 1988

URI : https://id.erudit.org/iderudit/027050ar

DOI : https://doi.org/10.7202/027050ar

Aller au sommaire du numéro

Éditeur(s)

Société de philosophie du Québec

ISSN

0316-2923 (imprimé)

1492-1391 (numérique)

Découvrir la revue

Citer cet article

Moggach, D. (1988). Phénoménologie et dialectique du travail. Philosophiques, 15(2), 311-329. https://doi.org/10.7202/027050ar

\section{Résumé de l'article}

Ludwig Landgrebe interprète les réductions phénoménolo- gique et eidétique de Husserl comme théorie de la corporéité, du travail et de la société, pour situer le sujet actif dans le monde naturel et historico-culturel. Cette théorie repose toujours sur un individualisme aprioriste. Une ontologie sociale, inspirée surtout des derniers ouvrages de Lukacs, cherche le principe de synthèse des dimensions concrètes et structurelles de l'expérience dans la logique dialectique du processus de travail lui-même, plutôt que dans la corporéité, et reformule ainsi le problème de la constitution transcendantale. 


\title{
PHÉNOMÉNOLOGIE ET DIALECTIQUE DU TRAVAIL
}

\author{
par Douglas Moggach
}

\begin{abstract}
RÉSUMÉ. Ludwig Landgrebe interprète les réductions phénoménologique et eidétique de Husserl comme théorie de la corporéité, du travail et de la sociéré, pour situer le sujet actif dans le monde naturel et historico-culturel. Cette théorie repose toujours sur un individualisme aprioriste. Une ontologie sociale, inspirée surtout des derniers ouvrages de Lukacs, cherche le principe de synthèse des dimensions concrètes et structurelles de l'expérience dans la logique dialectique du processus de travail lui-même, plutôt que dans la corporéité, et reformule ainsi le problème de la constitution transcendantale.
\end{abstract}

\begin{abstract}
Ludwig Landgrebe interprets Husserl's phenomenological and eidetic reductions as a theory of corporeality, labour and society in order to situate the active subject in the natural and historicocultural world. He remains committed, however, to an a priori individualism. A social ontology inspired by Lukacs's final writings seeks to synthesize the concrete and structural dimensions of experience not in corporeality as such, but in the dialectical logic of the labour process itself, thus giving a new formulation to the problem of transcendental constitution.
\end{abstract}

Dans la personne de son représentant le plus éminent, Edmund Husserl, le mouvement phénoménologique dans la philosophie contemporaine répond à la conscience aiguë d'une crise. Husserl propose une défense idéaliste de la raison et de l'autonomie contre la réification de la société moderne, la diminution de la personne au statut de chose. Dans cette société réifiée, la raison s'oublie et s'efface devant ses produits. ${ }^{1}$ La crise s'explique par

1. R. Miguelez, Sujet et histoire, Ottawa, 1973, p. 19 sq. ; et du même auteur, «La deuxième crise de la raison et la critique du socialisme ", Politique et raison, Figures de la modernité, Otrawa, 1988, p. 63-78. 
l'aliénation de la raison. ${ }^{2}$ Elle se scinde d'une part en pur calcul instrumental, limité aux techniques, qui renonce à la détermination des fins, et d'autre part en nihilisme irrationaliste qui nie toute aptitude directrice de la raison. Dans ces circonstances la tâche de la philosophie devient une défense multiple de la rationalité. Elle doit incarner les efforts millénaires de l'humanité vers la liberté rationnelle, mais maintenant elle est obligée de défendre ses acquis contre la tendance de la société actuelle. Elle doit conserver et protéger le sens profond de la civilisation contre une société qui supprime son propre potentiel. La philosophie doit souligner le rôle actif, constitutif de la raison dans la genèse du monde des valeurs culturelles; elle doit appeler à l'anamnesis, ${ }^{3}$ au souvenir d'une ouvre commune dans l'édification d'un monde humanisé. Enfin elle doit reconnaître sa propre responsabilité dans la crise actuelle; par une critique de ses propres prémisses elle doit renouveler la signification de la raison contre ses nouveaux adversaires. Elle doit restaurer le sens critique et libérateur de la raison comme principe fécond de la vie individuelle et collective.

À cette rationalité vécue Husserl oppose une autre vision, celle d'une "rationalisation" scientiste et techniciste du monde. C'est ici que débute sa conception de la crise. Il attaque l'extension illégitime de la science au-delà de ses propres limites, pour englober toutes les sphères de la vie humaine. À l'instar de Kant, il distingue entre un usage légitime et illégitime de la raison scientifique. La science transgresse ses limites méthodologiques quand elle oublie l'activité du sujet humain en faveur d'un pur objectivisme, qui se détache de son fondement dans la vie sociale et culturelle (Lebenswelt), qui défigure ces sphères à sa propre image, et qui s'arroge le statut de la seule vérité. La science transgresse ses limites éthiques quand elle oublie et réprime son projet originaire de libération, quand elle réduit l'être humain à un objet de manipulation ${ }^{4}$. Dès ses premiers écrits Husserl accentue le rôle créateur et actif de la subjectivité contre l'objectivisme, mais sa compréhension de la crise s'approfondit quand il constate que

2. E. HUSSERL, La crise de l'bumanité européenne et la philosopbie [Conférence de Vienne, 1935], édition bilingue avec une traduction de P. Ricceur, Paris, 1977, p. 103.

3. L. LANGREBE, "Life-World and the Historicity of Human Existence », in B. Waldenfels et al., Pbenomenology and Marxism, Londres, 1984, p. 175.

4. E. HuSSERL (1977), p. 43, 83. 
l'essor du fascisme - cette «zoologie des peuples " ${ }^{5}$ - menace de déchirer le tissu de la rationalité classique émancipatoire, dont il se déclare le partisan. ${ }^{6} \mathrm{~L}$ 'irrationalisme, si illusoirement nouveau et si superficiellement radical, n'est que le symptôme d'une nouvelle tournure, d'une exacerbation de la crise de la société moderne. Il faut donc combattre sur deux fronts : contre l'hypertrophie de la raison techniciste, avec ses conséquences de manipulation et de calcul instrumental; et contre l'irrationalisme moderne, qui conjugue les techniques de contrôle les plus avancées avec une idéologie barbare et rétrograde. ${ }^{7}$

Pour mener cette luttre il faut une reprise du principe originaire de la philosophie moderne, celle du cogito ou de la subjectivité. Ce sont précisément les formulations inadéquates de ce principe chez Galilée, Descartes et Kant, entre autres, qui ont affaibli la réponse de la philosophie à ses adversaires, et qui ont même défriché le terrain à partir duquel ses détracteurs lancent leurs attaques anti-philosophiques. ${ }^{8}$ Comment pouvons-nous repenser le rapport du sujet et du monde naturel et historicoculturel ? Une nouvelle justification de la raison peut se fonder sur une théorie de l'activité constitutive de la subjectivité et de l'intersubjectivité.

Dans la thématisation de ce domaine de problèmes, Husserl ouvre une voie que quelques-uns de ses disciples, notamment Ludwig Landgrebe, vont explorer comme théorie de la corporéité,

5. Ibid., P. 33.

6. L'importance du sous-texte de la Conférence de Vienne de Husserl a été soulignée par D. CARR, "Introduction", dans E. Husserl, The Crisis of European Sciences and Transcendental Phenomenology, trad. D. Carr, Evanston, Illinois, 1970, p. xxvi-xxvii. La critique de l'irrationalisme nazi est dispersée à travers la Conférence de Vienne : voir E. Husserl (1977), p. 31-3 (le rappel de l'Allemagne à son appartenance à Ia famille européenne, à la participation à son héritage rationaliste); p. 57-9 (contre les traditions irrationnelles) ; p. 61 (le rationalisme commun qui dépasse et qui englobe les nations) ; p. 73 (le terrain commun de l'objectivisme et de l'irrationalisme) et p. 103 (contre les idéologies de haine et de barbarie).

7. E. Husserl (1977), p. 103; E. Husserl, Die Krisis der europäischen Wissenschaften und die transzendentale Phänomenologie, Husserliana, Bd. VI, éd. W. Biemel, La Haye, 1962, p. 1,4

8. E. Husserl (1962), p. 74 sqq 
du travail et de la société. ${ }^{9}$ Dans cette nouvelle version elle indique la possibilité d'une convergence avec certains courants au sein du marxisme, qui examinent les mêmes questions du point de vue de la logique dialectique et qui partagent avec Husserl l'objectif de synthétiser les dimensions concrètes et structurelles de l'expérience humaine.

Pour Husserl lui-même toute concordance entre la phénoménologie et le marxisme serait exclue a priori. Des commentateurs ont déjà observé qu'il n'y a pas une seule référence à Marx dans tout le corpus husserlien. ${ }^{10}$ Il existe des raisons systématiques profondes pour ce silence. Lauer, par exemple, suggère qu'en tant que position essentialiste la phénoménologie est nécessairement anhistorique. Pour lui la méthode de Husserl consiste dans un mouvement qui part du concret pour aboutir à l'abstrait, une distanciation par rapport à l'histoire réelle, et une dévaluation du contingent et du factuel en faveur des essences permanentes «contenues » dans l'expérience. ${ }^{11}$ Dans la perspective de l'Ontologie de l'être social de Lukacs, l'approche husserlienne est aux antipodes du marxisme parce qu'elle pose la priorité de la logique sur l'histoire, parce qu'elle assimile le processus historique à des catégories formelles d'essence. ${ }^{12}$ Dans une étude des œuvres de jeunesse de Marcuse, Alfred Schmidt accentue, lui aussi, l'incompatibilité profonde entre le marxisme et la phénoménologie ; ici, bien sûr, dans sa ligne heideggerienne plutôt que husserlienne, mais en visant les éléments communs à la méthode phénoménologique. Tout au mieux, selon Schmidt, la tentative de synthèse résulte dans un dualisme entre les éléments immanents-formels et historiques, une dichotomie de la validité et de la genèse qui est typique de l'entreprise néo-kantienne. Elle crée un monde de sens, dérivés de la conscience pure, en juxtaposition maladroite avec un

9. Voir surtout L. LANGREBE, The Phenomenology of Edmund Husserl, trad. D. Welton, Ithaca, 1981; et ses deux articles dans B. Waldenfels et al., Phenomenology and Marxism, Londres, 1984 : «The Problem of Teleology and Corporeality in Phenomenology and Marxism", p. 53-81, et "Life-World and the Historicity of Human Existence ", p. 167-204.

10. Q. LAUeR, The Triumph of Subjectivity, 2e éd., New York, 1978, p. 169 n.

11. Ibid., p. $172 \mathrm{ff}$.

12. G. LuKacs, Zur Ontologie des gesellschaftlichen Seins, Erster Halbband, Werke, Bd. XIII, Darmstadt et Neuwied, 1984, p. 468-558. 
contenu historique. ${ }^{13}$ Adorno a également démontré l'incommensurabilité entre les prémisses du matérialisme historique et les éléments idéalistes et individualistes de la pensée de Husserl. ${ }^{14}$ Ces témoignages sont imposants, et nous ne devons pas les sousestimer. Mais il serait peut-être possible de répondre à ces critiques si on pouvait identifier des structures constitutives de la vie sociale qui contiennent en même temps des dimensions formelles-abstraites et concrètes-historiques, capables de montrer l'intersection entre les approches phénoménologique et dialectique. Cette interrogation pourrait nous amener à des conclusions apparemment paradoxales. Dans cette voie nous devons examiner d'abord des tensions fondamentales de la philosophie de Husserl qui pourraient valider l'élargissement de ses horizons de sens; ensuite nous pourrons étudier les enjeux des tentatives plus explicites de synthèse.

La procédure phénoménologique nous oblige à chercher, derrière le monde tel qu'il est constitué, les actes de sa genèse dans la conscience. ${ }^{15}$ Ceci implique une phénoménologie descriptive ou « mondaine », et une phénoménologie proprement transcendantale. La première décrit et situe les diverses sphères de l'existence dans leur spécificité : leurs frontières, leurs contours, le mode selon lequel elles se présentent à nous ${ }^{16}$, bref leur topologie. La deuxième ramène ces sphères à des types spécifiques d'activité de structuration, aux différentes modalités de la subjectivité transcendantale, pour démontrer comment elles sont constituées. Une procédure "eidétique » est maintenant activée pour illustrer le caractère permanent et intersubjectivement valable des résultats découverts.

Les prémisses de cette approche se dégagent dans l'affrontement critique avec les antagonistes les plus hétérogènes. Contre l'ontologie de type platonicien, Husserl veut montrer que l'expérience des phénomènes contient en soi des éléments qui sont absolument

13. A. SCHмidT, «Existential-Ontologie und historischer Materialismus bei Herbert Marcuse ", in J. Habermas (ed.), Antworten auf Herbert Marcuse, Frankfurt a.M., 1968, p. 41-49.

14. T. ADORNo, Zur Metakritik der Erkenntnistheorie, Studien zur Husserl und die phänomenologischen Antinomien, Frankfurt/M., 1970, p. 130-161, 222-235.

15. E. Husserl (1977), p. 97.

16. D. WeltoN, "Introduction: World and Consciousness», dans L. Landgrebe, The Phenomenology of Edmund Husserl, p. 26-7. 
nécessaires et universels. ${ }^{17} \mathrm{La}$ procédure qu' il appelle la réduction eidétique doit garantir précisément cette relation entre l'expérience et la nécessité. Mais il utilisera ce motif également contre des adversaires plus immédiats, contre l'objectivisme et la mathématisation de la science moderne, qui réduit la sensibilité à un élément purement relatif ; ${ }^{18}$ et contre le psychologisme de Dilthey qui survalorise ce relativisme. La doctrine husserlienne de l'intuition des universels à caractère nécessaire ${ }^{19}$ doit garantir que la nécessité réside dans l'expérience, et qu'elle soit subjective sans être réductible à la psychologie empirique. ${ }^{20}$

Alors que l'ontologie traditionnelle prétend examiner les choses et leur manière d'être séparées de la conscience subjective, Husserl interroge la manière de donation des phénomènes, leur rapport à la subjectivité. ${ }^{21} \mathrm{Il}$ suit la tournure kantienne envers les conditions de possibilité des objets. Ceci constitue le but de la réflexion phénoménologique. Contre Descartes et Kant, cependant, il insiste sur l'historicité du moi. L'explication cartésienne et critique de la subjectivité transcendantale n'est pas pure. Elle retient des éléments non analysés, non critiques de l'attitude naturelle naïve. Donc chez Descartes la mise entre parenthèses du monde révèle une simple opposition entre le sujet et l'objet, et une conscience individuelle non problématique, empirique. ${ }^{22}$ Dans ses Méditations cartésiennes (1929), Husserl essaie de développer une conception plus adéquate, voire plus dialectique de l'expérience, qui découvre la polarité, l'interdépendance des côtés subjectifs et objectifs dans la rencontre avec des phénomènes. Dans les années '30 sa conception de l'historicité de l'expérience s'élargit pour

17. A. PAZANIN, Wissenschaft und Geschichte in der Phänomenologie Edmund Husserls, La Haye, 1972, p. 115-18.

18. E. HuSSERL (1977), p. 17.

19. G. KüNG, "The Phenomenological Reduction as Epoche and as Explication», The Monist, vol. 59, $\mathbf{n}^{\circ} 1,1975$, p. 64-5. Cf. E. Husserl, «Der Weg durch die Kritik der positiven Wissenschaften zur transzendentalen Phänomenologie, der cartesianische Weg der Ideen und das Problem der vorgegebenen Lebenswelt », Husserliana, Bd. VIII, p. 259-274; et « Der cartesianische und der Weg der universalen phänomenologischen Psychologie in die transzendentale Phänomenologie", Husserliana, Bd. VIII, p. $275-301$.

20. E. HuSSERL (1977), p. 89-91.

21. A. PAZANin, op. cit., p. 4-5.

22. E. HuSSERl (1962), p. 74 sqq. 
inclure des dimensions culturelles et sociales de la Lebenswelt commune, ${ }^{23}$ peut-être sous l'influence de son disciple Landgrebe. ${ }^{24}$ Husserl souligne aussi l'ambiguïté de l'héritage de Descartes qui réoriente l'épistémologie vers la connaissance apodictique mais qui initie en même temps cet objectivisme selon lequel le monde mathématique des sciences naturelles est le seul monde véritable. ${ }^{25}$

Chez Kant aussi, l'idée d'un monde commun, partagé, et historiquement construit est insuffisamment élaborée. ${ }^{26}$ L' « Idée » kantienne du monde, à laquelle strictement aucune expérience ne correspond, doit céder sa place à l'expérience phénoménologiquement clarifiée de la Lebenswelt comme horizon intersubjectif du sens culturel et social. Il faut aussi éliminer ce qu'il y a d'empiriste et de psychologiste dans la doctrine kantienne de la perception. Cet objectif, annoncé dans les Recherches logiques et dans la Crise des sciences européennes et la pbénoménologie transcendantale, sera rempli explicitement dans les ouvrages de Landgrebe.

L'attitude naturelle que critique Husserl présume que le monde est antérieur au moi et indépendant du moi. Ce moi, conçu comme sujet naturel empirique, est partie intégrante du monde objectif, une chose parmi d'autres. ${ }^{27}$ Husserl veut plutôt montrer qu'il existe une source ultime de sens, de valeur, et même d'objectivité, cachée et oubliée derrière le voile de la réification qu'exemplifie l'attitude naturelle.

Cette source c'est la conscience transcendantale. Le monde est à révéler comme phénomène qui existe pour et par l'activité subjective. La méthode husserlienne vise à restaurer notre conscience de cette activité fondatrice occultée par le naturalisme naïf. Il veut réorienter notre attitude envers le monde, et reconstituer le sens

23. D. CARR, Phenomenology and the Problem of History, Evanston, Illinois, 1974, cité dans Welton, op. cit., p. 29.

24. D. Welton, op. cit., p. $28-9$.

25. E. HUSSERl (1962), p. 74-76.

26. Ibid., p. 101-113; Cf. E. Husserl, «Kants kopernikanische Umdrehung 》 (1924) et “ Kant und die Idee der Transzendental-philosophie " (1924), Husserliana, Bd. VII, La Haye, 1956, p. 208-229 et 230-287.

27. E. Husseri, Erste Philosophie (1923-24), Zweiter Teil, Husserliana, Bd. VIII, éd. R. Boehm, La Haye, 1959, p. 27, 44 sqq; M. Theunissen, Der Andere, Studien zur Sozialontologie der Gegenwart, Berlin, 1965, p. 30-31. 
de notre subjectivité. Il veut réduire, ramener le monde réifié à ses origines dans l'activité subjective, tout en libérant le concept du sujet de ses limitations « naturalistes » que la philosophie antérieure avait conservées. Il veut redécouvrir l'activité fondatrice de la raison et les déterminants rationnels et universels de l'activité : ses aspects phénoménologiques et eidétiques.

La procédure implique deux étapes distinctes, deux « réductions » séparées. La réduction phénoménologique ou transcendantale, dirigée vers la subjectivité empirique immédiate et sur le monde externe réifié des objets, révèle l'origine des formes de l'expérience dans l'activité subjective. Elle dévoile la conscience transcendantale active. Cette conscience n'est surtout pas à assimiler à la conscience empirique, mais elle est plutôt la base sur laquelle celle-ci s'édifie. Mais selon Husserl la conscience transcendantale est quand même fondamentalement individuelle. Comme le démontre Theunissen, la notion d'une subjectivité fondatrice n'est jamais transpersonnelle ou impersonnelle chez Husserl. Elle est toujours enracinée dans une «égologie », un moi individuel qui est par ailleurs strictement distingué de la conscience empirique. ${ }^{28}$ Elle ressemble peut-être davantage à une monade leibnizienne qui offre une perspective unique et irréductible sur le monde. Ce point a des implications importantes pour la possibilité d'une ontologie sociale. La relativité du monde par rapport à une subjectivité transcendantale individuelle semble exclure tout accès à l'intersubjectivité ou à une structure monadologique non dogmatique, mais l'intersubjectivité joue un rôle tout aussi fondamental chez Husserl. Voici une difficulté centrale dont Landgrebe va traiter.

La réduction transcendantale implique l'épochè, la mise entre parenthèses de la question ontologique de l'existence indépendante des choses, ou de la matière. Comme l'exprime Landgrebe, l'épochè fait surgir le sens en tenant en suspens la question de la validité des phénomènes. ${ }^{29} \mathrm{Si}$ oui ou non cette procédure engage Husserl à l'idéalisme ontologique, c'est une question qui a été passionnément débattue par les commentateurs. Husserl lui-même nie toute

28. M. TheunisSen, op. cit., p. 29.

29. L. LandGrebe, The Phenomenology of Edmund Husserl, p. 92, Cf. E. Husserl, Erste Philosophie Il, p. 92-112. 
attribution d'idéalisme subjectif à cette position ; ${ }^{30}$ en cela il est sourenu par Holmes. ${ }^{31} \mathrm{Il}$ faut remarquer aussi des tensions dans les différentes discussions de ce problème chez Husserl. Ricœur contraste le " réalisme » plus marqué des Idées $I^{32}$ avec la discussion plus idéaliste de la méthode dans les Méditations cartésiennes, mais il conclut finalement que Husserl confond le problème de l'Être avec la position naïve des objets particuliers dans l'attitude naturelle, qui omet la référence à la subjectivité. ${ }^{33}$ Adorno développe une critique similaire de l'identification idéaliste de la réification et de l'objectivation; il souligne en contraste l'irréductibilité de la matière à la pensée. ${ }^{34}$

L'autre élément dans le transcendantalisme husserlien c'est la réduction eidétique. Si la réduction phénoménologique révèle la relativité du monde à la conscience, il est nécessaire maintenant de poser l'unité de ce monde, de fournir le cadre non dogmatique pour l'accord des perspectives monadiques. Il s'agit des structures permanentes et intersubjectivement valables de l'expérience. Mais comment concilier la notion des essences partagées par tous les sujets avec les implications individualistes de la réduction phénoménologique? En effet, la relation des deux réductions reste obscure dans les ouvrages de Husserl. La procédure eidétique dérive de l'antipsychologisme et du transcendantalisme incomplet des Recherches logiques de sa jeunesse, ${ }^{35}$ et il n'a jamais réussi à exprimer sans ambiguïté le rapport mutuel des deux réductions. Néanmoins la fonction de l'eidos est claire. Il répond au problème platonicien de l'unité dans la diversité, avec la différence que maintenant les essences se situent dans la conscience subjective; elles sont les noyaux des phénomènes qui émergent dans l'expérience, dans l'intentionnalité. Voici une formulation tout à fait moderne du problème. Comme théorie de l'intersubjectivité elle vise le dépassement de cet idéalisme subjectif au singulier que Hegel a

30. E. HUSSERL, Ideen zu einer reinen Pbänomenologie und phänomenologischen Philosophie, Erstes Buch, Husserliana, Bd. III, La Haye, 1950, p. 134-136.

31. R.H. Holmes, "Is Transcendental Phenomenology Committed to Idealism? », The Monist, vol. 59, no 1,1975 , p. 98-114.

32. P. Ricaur, Husserl, an Analysis of his Phenomenology, Evanston, Ill., 1967, p. 88-9.

33. Ibid., p. 190.

34. T. ADORNO, op. cit., p. 215, 234-5.

35. M. THeunissen, op. cit., p. 37-38. 
depuis longtemps critiqué. L'eidos est également accessible et nécessaire à tous les sujets ; il fonde donc la rationalité et l'intersubjectivité, contre l'irrationalisme moderne dans toutes ses variantes.

Si l'analyse de l'eidos sépare les aspects contingents et particuliers de l'expérience et ses structures formelles permanentes, elle semble devoir exiger aussi la reconstitution du pôle subjectif. On pourrait s'attendre à ce que l'individualité du sujet transcendantal se réduise aussi à un moment transitoire et contingent séparé de son propre eidos, qui apparaîtrait maintenant comme intersubjectivité. Mais ce passage dialectique n'a pas lieu chez Husserl. C'est seulement dans un sens mitigé que le moi perd son individualité déterminée : l'eidos de la subjectivité est toujours dépeint comme potentialité transformationnelle du moi individuel. Il représente les variations que le moi effectue sur soi-même, l'éventail de ses propres possibilités individuelles. ${ }^{36} \mathrm{Il}$ faut conclure que Husserl n'arrive pas à une théorie satisfaisante des relations entre les sujets. L'intersubjectivité est dérivée d'un sujet primordial autonome et isolé, de «l'illusion bourgeoise » que Christopher Caudwell avait caractérisée avec tant d'élan. ${ }^{37}$ Car comment est-il possible que ces variations de soi individuelles coïncident? Ricour propose une solution qui souligne le contenu commun universel de l'eidos comme communauté rationnelle. Une sorte de monadologie leibnizienne intègre la perspective de chaque monade dans une structure formelle permanente. ${ }^{38}$ Mais pour éviter le dogmatisme de Leibniz il serait nécessaire que cette structure ne soit pas une forme pré-donnée sur laquelle les particuliers réfléchissent. Il faudrait qu'elle émerge à partir des actes des sujets. Cette formulation ne fait que transposer le problème de la concordance des subjectivités. Elle génère une antinomie : ou bien elle peut garantir l'unité de l'essence, mais au prix de son statut transcendantal; ou bien elle peut fonder l'eidos dans l'activité subjective, sans pourtant pouvoir démontrer le fondement possible de son unité. L'eidos intersubjectivement disponible constitue-t-il un élément pré-donné qui n'est pas réductible aux activités du sujet transcendantal ? Y a-t-il donc

36. Ibid., p. 40-44.

37. C. CaUdWEll, The Concept of Freedom, Londres, 1977, p. 51-75.

38. P. RICCEUR, op. cit., p. 137-42. 
un substrat encore plus caché qui n’a pas été clarifié phénoménologiquement? Il est fort possible que Husserl ne fournisse pas de solution adéquate à ce problème. Il dépeint une genèse fondamentalement individualiste de l'expérience.

Mais ici la voie est barrée à la communauté et à l'histoire. Alors que la théorie exige l'intersubjectivité, les conditions de son émergence restent invisibles. Cette difficulté a suscité de nombreuses critiques. Theunissen, par exemple, veut démontrer les implications solipsistes du transcendantalisme, qui propose de construire les autres sujets dans et par la subjectivité individuelle, et qui leur attribue un statut purement dérivé et nullement indépendant. 39 Dans sa défense du courant «dialogique » exemplifié par Buber, Theunissen surestime peut-être la technique de la réduction eidétique par la variation individuelle, plutôt que le caractère intersubjectivement valable de ses résultats. Selon Küng la question des autres esprits pose une difficulté plus marquée dans la procédure cartésienne que dans la méthode psychologique de la réduction phénoménologique. ${ }^{40}$ Mais il semble bien que ni l'alterité des sujets, ni celle de la nature ne soient garanties par l'approche husserlienne.

Le problème du temps et de l'histoire chez Husserl suscitent les critiques de Fink ${ }^{41}$ et de Pazanin. Selon ce dernier l'histoire devient invisible dans une théorie qui réduit le monde à des phénomènes spectraux qui confrontent un moi sans chair ni conscience de soi [ein fleischloses und ichloses Ich]. ${ }^{42}$ Dans sa défense de Husserl, Landgrebe passe en revue la gamme des critiques dans les publications allemandes récentes: la légitimité du glissement, non annoncé, de la coprésence du monde et du moi vers l'apodicticité du moi seul; le rapport entre la Lebenswelt individuelle et collective ; et la genèse individualiste et idéaliste de la société dans des actes de la conscience. ${ }^{43}$ Mais Landgrebe croit qu'il est possible de répondre à ces critiques par une réaffirmation de la réduction transcendantale contre des malentendus, et de

39. M. Theunissen, op. cit., p. 43. Cf. E. Husserl, Erste Philosophie II, p. 173-181.

40. G. KÜNG, op. cit., p. 67.

41. E. Fink, "Welt und Geschichte", Phaenomenologica, vol. 2, 1956, p. 152-3, cité d'après A. Pazanin, op. cit., p. 166-7.

42. A. PAZANin, op. cit., p. 143.

43. L. LANDGREBE, "Life-World and the Historicity of Human Existence», p. 169-73. 
fournir une nouvelle base pour la certitude du moi transcendantal qui ouvre en même temps vers l'intersubjectivité.

L'élaboration de la phénoménologie husserlienne par Landgrebe propose une interprétation de la subjectivité transcendantale comme corporéité, un nouveau concept de la perception et de l'activité kinésthésique, une dérivation pratique des catégories de l'espace et de la temporalité, et une nouvelle base pour l'intersubjectivité. Pour Landgrebe la corporéité devient la source transcendantale du sens. ${ }^{44}$ Dans son activité perceptuelle et dans son effectivité téléologique et transformatrice, le corps constitue le lien réel entre le moi, la nature, la communauté et l'histoire. Mais il faut surtout préserver le sens de l'activité transcendantale tout en représentant l'être matériel. Il faut donc donner une explication des activités du corps comme expérience immédiate vécue, non pas dans le médium de l'objectivisme réifiant, et il faut reconsidérer la source de cette expérience concrète. Comment est-il possible de garantir le caractère transcendantal du moi et son effectivité matérielle sans l'objectiver? La réponse réside dans l'unité dialectique du moi et du corps, ${ }^{45}$ identité dans la différence plutôt qu'une simple assimilation. ${ }^{46}$ Le moi n'est pas le corps, mais il y est indissociablement lié. Il se sert du corps, ${ }^{47}$ et dans cet usage il atteint une conscience immédiate et non réifiée de soi-même. L'activité corporelle du moi c'est le niveau constitutif qui est implicite dans les actes manifestes. ${ }^{48}$ Landgrebe pose également les bases d'une nouvelle théorie de la perception, qui n'est plus une réception passive des données mais un processus d'autodétermination dans l'expérience kinésthésique qui surgit dans la rencontre corporelle

44. L. LANDGrebe, «The Problem of Teleology and Corporeality in Phenomenology and Marxism \%, p. 64.

45. D. WELTON, op. cit., p. 26.

46. L. LANDGREBE, The Phenomenology of Edmund Husserl, p. 64.

47. D. WELTON, op. cit., P. 26-8.

48. Ibid., p. 19-20. Un autre engagement envers le transcendantal a priori a lieu chez Jürgen Habermas, Erkenntnis und Interesse, Frankfurt/M., 1968, et Theorie des kommunikativen Handelns, 2 vols., Frankfurt/M., 1981. La solution de Habermas a recours moins à l'idée de la corporéité elle-même qu'aux conditions nécessaires et universelles de l'action, qui sont à découvrir et à démontrer à l'aide des sciences empiriques, a posteriori. Voir R.J. Bernstein, «Introduction », in R.J. Bernstein (éd.), Habermas and Modernity, Cambridge, Mass., 1985, p. 13, 16-19. 
du moi avec le monde. ${ }^{49}$ Landgrebe suit une démarche fichtéenne pour dériver de la pratique les catégories perceptives. La temporalisation et la spatialisation sont des processus actifs du sujet qui affronte et qui assimile la résistance de la matière. ${ }^{50}$ Dans cette synthèse de perception et de téléologie, le sujet s'ouvre vers la communauté et l'histoire. Selon Landgrebe la temporalité et la corporéité sont la base d'une théorie de l'historicité. 51 Tout comme l'Endzweck kantien, nous pouvons comprendre l'histoire seulement à condition que nous y posions un principe téléologique. Ce principe n'est ni un fait empiriquement donné [gegenwärtig wirkliches], ni un eschaton métaphysique, mais c'est le principe de la structure temporelle de l'être et de l'action humaine, l'activité téléologique qui réalise ses fins dans le concret. ${ }^{52}$ C'est ce principe qui est déjà implicite dans l'activité perceptive.

Selon Landgrebe la corporéité et l'action kinésthésique résolvent également le problème de l'intersubjectivité chez Husserl. Elles démontrent la singularité du monde, la manière selon laquelle chaque individu vise son monde; et aussi l'invariant de tous les mondes, la commensurabilité d'une structure commune qui sert de base pour l'initiation de la communication. ${ }^{53} \mathrm{Il}$ affirme :

In this sense we can make the compatibility of the two determinations of the life-world as "concrete universality" and "universe of intuitability" intelligible : every world is intrinsically concrete universality, but it is a "universe of intuitability" with reference to that which makes it comparable with orher worlds. ${ }^{54}$

Remarquons bien cette formulation: l'universalité concrète appartient explicitement à l'expérience individuelle, à chaque monde singulier ; la possibilité du social réside dans la comparaison

49. L. Landgrebe, «The Problem of Teleology and Corporeality in Phenomenology and Marxism », p. 53-81.

50. L. Landgrebe, The Pbenomenology of Edmund Husserl, p. 34. Voir aussi A. Pazanin, op. cit., p. 171. Comparer l'affirmation de Husserl (1977), p. 19, sur le corps comme fondement de l'intersubjectivité, avec son observarion, p. 97, sur la subjectivité transcendantale comme spectateur théorique : Landgrebe insiste sur le caractère actif de la constitution.

51. L. LANDGREBE, Phänomenologie und Geschichte, Gürersloh, 1968, p. 7, 183-97, cité d'après A. Pazanin, op. cit., p. 158, 170-1.

52. Ibid., d'après A. Pazanin, op. cit., p. 170-1.

53. L. LANDGREBE, "Life-World and the Historicity of Human Existence», p. 184-6.

54. Ibid., p. 187 
des structures téléologiques-kinésthésiques de ces mondes. Tout comme Husserl, Landgrebe accorde une priorité théorique au sujet individuel. Les conditions de possibilité de la société sont toujours inscrites dans une égologie. En plus, l'invariant de ces mondes particuliers, ce sont la corporéité et ses activités kinésthésiques de perception, et non pas les structures du procès de travail ou la transformation active de la nature. Conçue comme détermination active du sujet, la perception constitue le paradigme eidétique de l'activité.

Mais nous ne pouvons pas résoudre les problèmes de l'intersubjectivité par une simple traduction du langage de Husserl dans un lexique plus matérialiste de corporéité, comme le propose Landgrebe. Sa théorie continue à affirmer le primat ontologique du sujet et de sa conscience sur la collectivité. La transposition du moi transcendantal en sujets concrets actifs est une condition nécessaire mais insuffisante pour théoriser le processus de travail. Le terrain possible de convergence est plus profond et requiert de repenser dans leur totalité les réductions transcendantale et eidétique.

C'est sur la base du caractère double du processus de travail, en même temps concret et abstrait, que nous pouvons envisager un parallélisme entre la phénoménologie et l'analyse historicomatérialiste. Le processus de travail est précisément cette activité constitutive de la vie sociale qui synthétise les dimensions historiques et formelles, tel que l'exige notre examen de Husserl. Il répond aux critères de la coprésence des éléments concrets et abstraits, et il permet une nouvelle formulation du problème de la genèse de la subjectivité, de la constitution de la société, et du rapport avec la nature externe. Il répond aussi aux critiques déjà énumérées de la possibilité d'une synthèse entre la phénoménologie et le marxisme. Le mouvement du concret vers l'abstrait doit se compléter par un mouvement dans le sens inverse, la reconstitution du concret par l'analyse de ses formes de structuration. Ces formes sont celles de l'action elle-même, et comme telles, elles ne sont pas statiques et purement analytiques, mais dynamiques et dialectiques. Et l'étude de ces formes, en permettant la reconstruction théorique du social et de l'historique, permet le dépassement des prémisses idéalistes et individualistes de la phénoménologie husserlienne. La réduction 
du monde à sa source dans l'activité subjective nous révèle maintenant le travail.

Marx lui-même accentue la double dimension du procès de travail. Il est concret et qualitatif ; il produit des valeurs d'usage par des formes spécifiques déterminées de l'activité. Mais il est simultanément abstrait et universel. Derrière toutes les formes du travail concret, et instancié dans chacune de ses réalisations, se trouve le pur travail universel ou social, la dépense indifférenciée des énergies humaines dans la transformation de la nature.

La dialectique de Marx saisit cette dimension abstraite du procès de travail comme exemplification simple des catégories de la dialectique elle-même. Nous pouvons lire les descriptions du travail dans les Grundrisse comme transposition matérialiste de la doctrine de la téléologie que développe Hegel dans la Science de la logique. ${ }^{55}$ Les moments du procès de travail, malgré son immédiateté et sa concrétude, sont des déterminations abstraites et générales dont la forme sociale n'est pas encore spécifiée. Il s'agit d'un rapport de « connexion simple " ${ }^{56}$ entre des éléments absolument universels: la substance ou substrat de l'activité, soit brut (la matière première), soit déjà modifié (les instruments de travail); l'activité transformatrice elle-même ; et le produit de cette activité. Le moment de la substance dépeint les conditions naturelles et instrumentales de l'activité, y compris la causalité efficiente. C'est l'être qui est le simple, le déjà-donné, l'originairement-indéterminé 57 qui contient la potentialité de recevoir la forme, d'être déterminé,

55. K. MARX, Grundrisse der Kritik der politischen Ökonomie (Robentwurf), Berlin, 1954, p. 24-25, 204, 264 sqq. L'appropriation marxiste de ces catégories est possible parce que Hegel prend déjà le travail comme modèle de son analyse. Voir G.W.F. Hegel, Wissenschaft der Logik, II, Werke, Bd. VI, Frankfurt/M., 1969, p. 436-461. Le travail abstrait, base de la commensurabilité des valeurs d'échange dans le capitalisme, exprime le travail social par la figure du travail individuel généralisé, donc dans une forme directement antithétique. Cf. K. Marx, Contribution à le critique de l'économie politique, trad. M. Husson et G. Badia, Paris, 1957, p. 10-12. Néanmoins le travail abstrait et le travail social sont identiques dans le sens qu'ils expriment le travail comme l'essence de laquelle tous les travaux concrets sont des manifestations.

56. H.S. Harris, «Notes», dans G.W.F. Hegel, The Jena System, 1804-5: Logic and Metaphysics, trad. J. Burbidge et G. di Giovanni, Kingston, 1986. p. 4n.

57. Il s'agit ici d'un manque relatif de détermination par rapport à une forme ontologiquement supérieure ou plus concrète. Voir Aristote, Métaphysique, livre Z; et K. Marx, "Critique du programme de Gotha ", Euvres choisies, Moscou, 1975, p. 327. 
mais qui est aussi la puissance de réaliser la forme, de la rendre substantielle. Le moment négatif d'activité ou de détermination représente la téléologie comme processus transformationnel, synthèse mobile des pôles subjectif et objectif : l'intentionnalité subjective qui se cristallise dans un objet, la négativité qui se manifeste dans le concret. C'est une potentialité négative qui s'affirme contre et dans la matière comme fin à réaliser, et qui en s'actualisant effectue un changement dans la nature en collaborant avec les lois implicites de la causalité. Dans cette interaction elle est cristallisée, déterminée dans une forme matérielle ou une valeur d'usage ; elle apparaît sous la forme du travail concret sans y être épuisée. Simultanément la nature est transformée, étant imprégnée de la forme tout en cédant sa substance matérielle à la force de travail et au produit, qui apparaît donc comme résultat de la substantialisation de la forme et la négation de l'immédiateté de la nature.

Cette structure, simple dans son universalité et dans la relation indéterminée de ses parties, c'est l'eidos contenu dans chaque travail concret particulier. Le passage à un niveau plus concret de la dialectique, le rapport entre le travail et la production, développe les implications de cette approche pour une théorie de l'intersubjectivité. Cette dialectique révèle que le travail n'est pas d'abord une activité purement individuelle, ni une relation technique directe à la nature qui n'est intégrée que plus tard dans une matrice sociale. Le travail et la production représente plutôt des niveaux particuliers de concrétude, chacun avec sa structure et sa fonction spécifique, qui permettent ensemble de saisir le processus historique comme l'évolution des formes de la communauté en interaction métabolique avec la nature.

Malgré l'argument selon lequel les deux modèles de travail et de production sont incompatibles et même antagonistes, ${ }^{58}$ notre lecture des Grundrisse et du Capital suggère que le travail et la production se lient dans un passage dialectique qui implique des niveaux d'abstraction décroissante et de complexité croissante. ${ }^{99}$

58. A. Heller, «Paradigm of Work - Paradigm of Production», in The Power of Shame, a Rational Perspective, Londres, 1985, p. 57-70.

59. Cf. M.J. PetrY, «Introduction», dans G.W.F. Hegel, Pbilosophy of Nature, vol. I, Londres, 1970, p. 78, 90. 
Le mouvement de l'essence vers le phénomène, du travail à la production et, au-delà, vers la diversiré empirique des formes historiques, est effectué théoriquement dans ce mouvement de l'abstrait vers le concret. C'est un processus par lequel une série de présupposés initiaux, de caractère abstrait, est progressivement abrogée pour que la richesse des médiations entre les phénomènes se manifeste de plus en plus clairement. ${ }^{60}$

Du point de vue de l'histoire de la philosophie, la dialectique du travail et de la production représente une version matérialiste de la logique du Philèbe de Platon, le mouvement d'un simple êtreensemble vers la connexion déterminée. ${ }^{61}$ Elle intègre à ce passage la doctrine aristotélicienne de la causalité. Elle décrit le mouvement de complexification où les synthèses antérieures, ontologiquement moins riches, de la matière et de la forme constituent une simple matière qui est absorbée dans une forme supérieure. Dans ses analyses du travail Marx élabore cette méthode, qui est déjà en évidence dans la Logique de Iéna de Hegel. Le premier niveau, le plus abstrait, est celui du processus de travail, dont les éléments conditions, activité, produit - sont absolument universels et absolument simples dans leur articulation. Soulignons bien qu'il ne s'agit pas ici d'une relation a priori individualiste ni technique envers la nature. Il s'agit de la structure logique de l'action ellemême.

Le processus de production représente une étape plus déterminée, plus concrète, et plus complexe. Il lie le procès général et abstrait de travail à un processus spécifique d'appropriation. Il situe ainsi de façon définie le travail dans la société. La relation simple et générale de l'activité à ses conditions est maintenant médiatisée par une forme sociale spécifique. Le réseau des relations sociales intervient entre l'activité et ses conditions naturelles et instrumentales pour déterminer la façon dans laquelle les travailleurs auront accès aux moyens de production que commande la société. La distribution sociale des conditions du travail est le premier sens de l'appropriation, dont en découle un deuxième, la distribution sociale des produits. En conséquence du contrôle exercé sur les

60. Cette méthode a aussi des affinités avec celle de David Ricardo. Voir R. Meek, Studies in the Labour Theory of Value, Londres, 1956, p. 93.

61. H.S. HARRIS, op. cit., p. 4. 
conditions du travail, les relations sociales interviennent aussi pour allouer le produit de l'activité parmi les producteurs et entre les producteurs et les non-producteurs. C'est dans cette synthèse du travail et de l'appropriation que l'identité historique du travailleur est déterminée, à savoir si le procès de travail apparaît comme l'activité d'individus isolés ou dans diverses formes de collectivité. ${ }^{62}$

À la fin de l'analyse nous redécouvrons le travail concret situé dans le cadre d'une forme de production sociale spécifique. Il n'est plus cette immédiateté qui constituait le point de départ de l'examen, mais il est transmué par son passage à travers l'abstraction. Le concret n'est plus l'immédiat, mais le hautement déterminé, riche d'articulation historique.

La complémentarité possible entre le dialectique et la phénoménologie est donc enracinée dans un principe fondamental du marxisme, la dualité du processus de travail ; mais réciproquement, l'analyse marxiste ainsi conçue répond à des motifs profonds de la pensée de Husserl. Il n'est pas simplement question de matérialiser le moi transcendantal sans remettre en question son individualité, sans reconsidérer les modes de son interaction avec la société et la nature. La réduction transcendantale nous ramène plutôt au travail concret ; la réduction eidétique indique une essence structurelle qui se révèle comme les catégories de la logique dialectique du procès de travail abstrait. Cette essence n'est pas une structure formelle simple, mais elle contient la contradiction, la négativité et le mouvement. Enfin, le sujet que la réduction eidétique découvre comme intersubjectivité trouve son identité historique façonnée dans la dialectique du travail et de l'appropriation. La médiation active de la nature et de la société est le terrain où émergent les formes historiques de la subjectivité.

62. F. ToKel, Zur Theorie der Gesellschaftsformen, Budapest, 1977, p. 27-79. 
La réduction phénoménologique des formes du monde nous ramène à une activité fondatrice et productive, au travail dans sa concrétude et dans l'abstraction nécessaire de ses structures dialectiques. Dans le jeu des dimensions abstraites et concrètes, la phénoménologie et le marxisme s'ouvrent réciproquement sur un plan théorique central, lieu de convergence possible. Il s'agit bien sûr d'une phénoménologie profondément modifiée, mais des problèmes dans la structure de la doctrine elle-même, et certaines de ces tendances actuelles, invitent les modifications nécessaires. Sur cette base une synthèse authentique et fructueuse serait encore possible. Les deux courants collaborent en refondant le point de départ de la théorie. La réponse à la question transcendantale de Descartes n'est pas que «je pense», mais que «nous travaillons ». 63

Faculté des sciences sociales

Université d'Ottawa

63. Nous pouvons poursuivre l'analyse aux niveaux encore plus concrets du procès de reproduction et de mode de production. Le procès de production a encore ceci d'abstrait, qu'il considère l'activité comme un seul acte, non pas comme cycle interconnecté d'actes. C'est ce présupposé qui est abrogé au troisième niveau, le procès de reproduction. Maintenant le produit de l'activité est alloué de façon déterminée pour la reproduction de la force de travail et des instruments de travail, lesquels réapparaissent renouvelés au début d'un nouveau cycle de production. En plus, au-delà du niveau de reproduction simple ou à une échelle constante, une portion du produit social peut apparaître comme surplus. La question cruciale de la reproduction et de la transformation, c'est le volume er la disposition du surplus : sa consommation productive qui permettra une expansion de la masse de travail disponible et de ses instruments, et donc une reproduction élargie; ou bien sa consommation improductive; son allocation différentielle entre diverses fractions de la classe ou des classes dominantes, ou le degré de sa rétention par les producteurs directs.

Cette conception permet la transition au concept encore plus concret de mode de production. Si le procès de production est l'unité dialectique du procès de travail abstrait avec un réseau formel d'appropriation, la base matérielle du mode de production est la notion dialectiquement plus riche du processus de reproduction, à laquelle le processus d'appropriation est juxtaposé en unité dynamique.

L'élaboration de ce modèle aura lieu ailleurs. 